\title{
On the Determinant of a Product of Two Polynomial Matrices
}

\author{
G. Ramesh ${ }^{1}$, P. N. Sudha ${ }^{2}$ \\ ${ }^{I}$ (Associate Professor of Mathematics, Govt Arts College (Auto), Kumbakonam,Tamil Nadu, India.) \\ ${ }_{2}^{2}$ (Assistant Professor of Mathematics, Periyar Maniammai University, Thanjavur, Tamil Nadu, India.)
}

Abstract: The Cayley Hamilton theorem for an arbitrary matrix $A$ is generalized to a polynomial matrix. It is proved that if $h(\lambda)=\operatorname{det}[f(\lambda) g(\lambda)]$ then $h(A)=0$ iff $\operatorname{det} f(A)=0$ or $\operatorname{det} g(A)=0$.

Keywords: Polynomial matrix, Hermitian, Skew Hermitian, Symmetric and Skew symmetric polynomial matrices.

\section{Introduction}

In England, J.J.Sylvester first introduced the term "Matrix" in the year 1848. Matrix algebra was nurtured by the work of Arthur Cayley in 1855. Matrices are vital and essential part of the area of mathematics. Applications of matrices are found in most of the scientific fields.

The Cayley Hamilton theorem is one of the most important theorem in Matrix analysis which is extremely versatile and useful. It was given by Cayley in his 1858 Memoir on the Theory of Matrices. It says that every square matrix satisfies its own characteristic equation $|A-\lambda I|=0$.

Early in the $\operatorname{development}$ the formula $\operatorname{det}(\mathrm{AB})=\operatorname{det}(\mathrm{A}) \operatorname{det}(\mathrm{B})$ provide a connection between matrix algebra and determinants.

The Cayley-Hamilton theorem and its generalizations have been used in control systems [2] and also au-tomation and control in [3], electronics and circuit theory [4], time-systems with delays [5], singular 2-D linear systems [6], 2-D continuous discrete linear systems [7], automation and electrotechnics [8], etc.

In this paper a generalization of the Cayley Hamilton theorem for polynomial matrices is presented. The linear polynomial matrix $(\mathrm{A}-\lambda \mathrm{I})$ of $\operatorname{det}(\mathrm{A}-\lambda \mathrm{I})$ in the classical Cayley Hamilton theorem is replaced by the general polynomial matrix $f(\lambda)=A_{0}+A_{1} \lambda+\ldots . .+A_{n} \lambda^{n}$ where $A_{i}^{\prime}$ s for $\mathrm{i}=0,1,2, \ldots, \mathrm{n}$ are square matrices of the same order.

It is proved that if $\mathrm{h}(\lambda)=\operatorname{det}[\mathrm{f}(\lambda) \mathrm{g}(\lambda)]$ then for a square matrix $\mathrm{A}, \mathrm{h}(\mathrm{A})=0$ iff $\operatorname{det} \mathrm{f}(\mathrm{A})=0$ or $\operatorname{det} \mathrm{g}(\mathrm{A})=0$. It is illustrated with help of some examples.

\section{Definition 2.1}

\section{Preliminaries}

A matrix A whose entries are polynomials is said to be a polynomial matrix.

Example 2.2

A $3 \times 3$ polynomial matrix of degree 2 is given below.

$$
A=\left(\begin{array}{ccc}
1 & \lambda^{2} & \lambda \\
0 & 2 \lambda & 2 \\
3 \lambda+2 & \lambda^{2}-1 & 0
\end{array}\right)=A_{0}+A_{1} \lambda+A_{2} \lambda^{2}
$$

$$
\text { where } A_{0}=\left(\begin{array}{ccc}
1 & 0 & 0 \\
0 & 0 & 2 \\
2 & -1 & 0
\end{array}\right), A_{1}=\left(\begin{array}{lll}
0 & 0 & 1 \\
0 & 2 & 0 \\
3 & 0 & 0
\end{array}\right) \text { and } A_{2}=\left(\begin{array}{lll}
0 & 1 & 0 \\
0 & 0 & 0 \\
0 & 1 & 0
\end{array}\right)
$$

Example 2.3

A $3 \times 3$ polynomial matrix of degree 2 with coefficient matrices are symmetric is given below.

$$
A=\left(\begin{array}{ccc}
1+2 \lambda & 2+\lambda+2 \lambda^{2} & 6+5 \lambda+\lambda^{2} \\
2+\lambda+2 \lambda^{2} & 4+4 \lambda+4 \lambda^{2} & 3+2 \lambda+3 \lambda^{2} \\
6+5 \lambda+\lambda^{2} & 3+2 \lambda+3 \lambda^{2} & 5+5 \lambda+\lambda^{2}
\end{array}\right)=A_{0}+A_{1} \lambda+A_{2} \lambda^{2}
$$




$$
\text { where } A_{0}=\left(\begin{array}{lll}
1 & 2 & 6 \\
2 & 4 & 3 \\
6 & 3 & 5
\end{array}\right), A_{1}=\left(\begin{array}{lll}
2 & 1 & 5 \\
1 & 4 & 2 \\
5 & 2 & 5
\end{array}\right) \text { and } A_{2}=\left(\begin{array}{lll}
0 & 2 & 1 \\
2 & 4 & 3 \\
1 & 3 & 1
\end{array}\right) \text {. }
$$

Example 2.4

A $3 \times 3$ polynomial matrix of degree 2 with coefficient matrices are skew symmetric is given below.

$$
A=\left(\begin{array}{ccc}
0 & 2-\lambda^{2} & 1+\lambda+5 \lambda^{2} \\
-2+\lambda^{2} & 3 \lambda & 3+2 \lambda+2 \lambda^{2} \\
-1-\lambda-5 \lambda^{2} & -3-2 \lambda-2 \lambda^{2} & 5 \lambda
\end{array}\right)=A_{0}+A_{1} \lambda+A_{2} \lambda^{2}
$$

where $A_{0}=\left(\begin{array}{ccc}0 & 2 & 1 \\ -2 & 0 & 3 \\ -1 & -3 & 0\end{array}\right), A_{1}=\left(\begin{array}{ccc}1 & 0 & 1 \\ 0 & 3 & 2 \\ -1 & -2 & 5\end{array}\right)$ and $A_{2}=\left(\begin{array}{ccc}0 & -1 & 5 \\ 1 & 0 & 2 \\ -5 & -2 & 0\end{array}\right)$.

Example 2.5

A $2 \times 2$ polynomial matrix of degree 2 with coefficient matrices are Hermitian is as follows

$$
A=\left(\begin{array}{cc}
1+\lambda^{2} & (1-i)-i \lambda+(2-i) \lambda^{2} \\
(1+i)+i \lambda+(2+i) \lambda^{2} & 3+\lambda+5 \lambda^{2}
\end{array}\right)=A_{0}+A_{1} \lambda+A_{2} \lambda^{2}
$$

where $A_{0}=\left(\begin{array}{cc}0 & 1-i \\ 1+i & 3\end{array}\right), A_{1}=\left(\begin{array}{cc}0 & -i \\ i & 1\end{array}\right)$ and $A_{2}=\left(\begin{array}{cc}1 & 2-i \\ 2+i & 5\end{array}\right)$.

Example 2.6

A $2 \times 2$ polynomial matrix of degree 2 with coefficient matrices are skew Hermitian is as follows

$$
A=\left(\begin{array}{cc}
i & (1-i)+i \lambda+(2-i) \lambda^{2} \\
(-1-i)-i \lambda+(-2+i) \lambda^{2} & 3 i
\end{array}\right)=A_{0}+A_{1} \lambda+A_{2} \lambda^{2}
$$

where $A_{0}=\left(\begin{array}{cc}i & 1-i \\ -1-i & 3 i\end{array}\right), A_{1}=\left(\begin{array}{cc}0 & i \\ -i & 0\end{array}\right)$ and $A_{2}=\left(\begin{array}{cc}0 & 2-i \\ -2+i & 0\end{array}\right)$

Lemma 3.1

\section{Main Theorem}

If $\mathrm{A}$ and $\mathrm{B}$ are square matrices of order $\mathrm{n}$ having elements as polynomials in $\lambda$ each of degree $\leq \mathrm{m}$ then the elements of the matrix $\mathrm{AB}$ are also polynomials in $\lambda$ degree $\leq \mathrm{mn}$.

Example 3.2

$$
\text { Let } A=\left(\begin{array}{cc}
1 & \lambda \\
\lambda^{2} & 0
\end{array}\right) \text { and } B=\left(\begin{array}{ll}
\lambda^{2} & 0 \\
\lambda & 1
\end{array}\right) \text { then } A B=\left(\begin{array}{cc}
2 \lambda^{2} & \lambda \\
\lambda^{4} & 0
\end{array}\right)
$$

Theorem:3.3

Let $f(\lambda)=A_{0}+A_{1} \lambda+\ldots . .+A_{m} \lambda^{m}$ and $g(\lambda)=B_{0}+B_{1} \lambda+\ldots . .+B_{m} \lambda^{m}$ be polynomial matrices for $f(\lambda), g(\lambda) \in M_{n}(F[\lambda])$ where $A_{i}^{\prime} s, B_{i}^{\prime} s \in M_{n}(F)$ for $\mathrm{i}=1,2,3, \ldots \mathrm{m}$ are square matrices of order $\mathrm{n}$ over the field F. If $\mathrm{h}(\lambda)=\operatorname{det}[\mathrm{f}(\lambda) \mathrm{g}(\lambda)]$, then $\mathrm{h}(\mathrm{A})=0$ if and only if $\operatorname{det} \mathrm{f}(\mathrm{A})=0$ (or) $\operatorname{det} \mathrm{g}(\mathrm{A})=0$.

Proof:

Let $\mathrm{h}(\mathrm{A})=0$. We have to prove that $\operatorname{det} \mathrm{f}(\mathrm{A})=0$ (or) $\operatorname{det} \mathrm{g}(\mathrm{A})=0$.

Assume the contrary that $\operatorname{det} \mathrm{f}(\mathrm{A}) \neq 0$ and $\operatorname{det} \mathrm{g}(\mathrm{A}) \neq 0$.

Since for any two square matrices $\mathrm{A}$ and $\mathrm{B}$, we have 


$$
\operatorname{det}(\mathrm{AB})=\operatorname{det} \mathrm{A} \operatorname{det} \mathrm{B}
$$

using (3.1), we have $\operatorname{det}(f(\lambda) g(\lambda))=\operatorname{det} f(\lambda) \operatorname{det} g(\lambda)$.

$$
\mathrm{h}(\mathrm{A})=\operatorname{det}[\mathrm{f}(\mathrm{A}) \mathrm{g}(\mathrm{A})]=\operatorname{det} \mathrm{f}(\mathrm{A}) \operatorname{det} \mathrm{g}(\mathrm{A}) \neq 0 \text {. }
$$

Which is a contradiction to our assumption that $h(A)=0$.

Hence $\operatorname{det} \mathrm{f}(\mathrm{A})=0$ (or) $\operatorname{det} \mathrm{g}(\mathrm{A})=0$.

Conversely,

Since

Let us assume that $\operatorname{det} f(A)=0$ (or) $\operatorname{det} g(A)=0$. We have to prove that $h(A)=0$.

$$
f(\lambda)=A_{0}+A_{1} \lambda+\ldots . .+A_{m} \lambda^{m} \text { and } g(\lambda)=B_{0}+B_{1} \lambda+\ldots . .+B_{m} \lambda^{m}
$$

are matrices of order $\mathrm{n} x \mathrm{n}$ having elements as polynomials in $\lambda$ each of degree $\leq \mathrm{m}$, therefore by lemma 3.1, we have

$$
f(\lambda) g(\lambda)=C_{0}+C_{1} \lambda+C_{2} \lambda^{2}+\ldots \ldots+C_{m n} \lambda^{m n}
$$

also det $\mathrm{f}(\lambda)$ and $\operatorname{det} \mathrm{g}(\lambda)$ are polynomials in $\lambda$ over $\mathrm{F}[\lambda]$ of degree $\leq m n$.

Using lemma 1 of [1], we have

$$
\operatorname{det} f(\lambda)=Q_{0}+Q_{1} \lambda+\ldots . .+Q_{m n} \lambda^{m n}
$$

and

$$
\operatorname{det} g(\lambda)=R_{0}+R_{1} \lambda+\ldots . .+R_{m n} \lambda^{m n}
$$

Also $\mathrm{h}(\lambda)=\operatorname{det}[\mathrm{f}(\lambda) \mathrm{g}(\lambda)]$ is a polynomial in $\lambda$ over $\mathrm{F}[\lambda]$ of degree $\leq 2 m n$.

Using lemma 1 of [1], we have

$$
\mathrm{h}(\lambda)=\operatorname{det}[\mathrm{f}(\lambda) \mathrm{g}(\lambda)]=P_{0}+P_{1} \lambda+P_{2} \lambda^{2}+\ldots \ldots+P_{2 m n} \lambda^{2 m n}
$$

Now using (3.1), we have

Using (3.3), (3.4) and (3.6) in (3.7)

$$
\operatorname{det}(f(\lambda) g(\lambda))=\operatorname{det} f(\lambda) \operatorname{det} g(\lambda)
$$

$$
\left(P_{0}+P_{1} \lambda+P_{2} \lambda^{2}+\ldots \ldots+P_{2 m n} \lambda^{2 m n}\right)=\left(Q_{0}+Q_{1} \lambda+\ldots . .+Q_{m n} \lambda^{m n}\right)\left(R_{0}+R_{1} \lambda+\ldots . .+R_{m n} \lambda^{m n}\right)
$$

Comparing coefficients of the like terms on both sides of equation (3.8), we get

$$
\left.\begin{array}{l}
Q_{0} R_{0}=P_{0} \\
Q_{0} R_{1}+Q_{1} R_{0}=P_{1} \\
Q_{0} R_{2}+Q_{1} R_{1}+Q_{2} R_{0}=P_{2} \\
\cdot \\
\cdot \\
\cdot \\
Q_{m n} R_{m n}=P_{2 m n}
\end{array}\right\} .
$$

Multiplying the equations in (3.9) by the matrices I, A, $\mathrm{A}^{2}, \mathrm{~A}^{3}$, $\mathrm{A}^{\mathrm{m}}, \mathrm{A}^{\mathrm{m}+1}$ $\mathrm{A}^{2 \mathrm{mn}-1}, \mathrm{~A}^{2 \mathrm{mn}}$ respectively and adding, we get

$$
\begin{aligned}
& Q_{0} R_{0} I+\left(Q_{0} R_{1}+Q_{1} R_{0}\right) \mathrm{A}+\left(Q_{0} R_{2}+Q_{1} R_{1}+Q_{2} R_{0}\right) \mathrm{A}^{2}+\ldots \ldots \\
& \ldots . .+\left(Q_{m n} R_{m n}\right) \mathrm{A}^{2 \mathrm{mn}}=P_{0} I+P_{1} A+P_{2} A^{2}+\ldots \ldots+P_{2 m n} A^{2 m n} . \\
& \Rightarrow\left(Q_{0} I+Q_{1} A+Q_{2} A^{2}+\ldots . .+Q_{m n} A^{m n}\right)\left(R_{0} I+R_{1} A+R_{2} A^{2}+R_{3} A^{3}+\ldots \ldots+R_{m n} A^{m n}\right)=h(A) \\
& \Rightarrow \mathrm{h}(\mathrm{A})=0 .
\end{aligned}
$$

Example3.4

Consider the function $f(\lambda)=A_{0}+A_{1} \lambda+A_{2} \lambda^{2}$;

where $A_{0}=\left(\begin{array}{ll}1 & 0 \\ 0 & 0\end{array}\right) ; A_{1}=\left(\begin{array}{ll}0 & 1 \\ 0 & 1\end{array}\right)$ and $A_{2}=\left(\begin{array}{ll}0 & 0 \\ 1 & 0\end{array}\right) \Rightarrow f(\lambda)=\left(\begin{array}{cc}1 & \lambda \\ \lambda^{2} & 1+\lambda\end{array}\right)$.

And $g(\lambda)=B_{0}+B_{1} \lambda+B_{2} \lambda^{2}$ 
where $B_{0}=\left(\begin{array}{ll}0 & 1 \\ 0 & 0\end{array}\right) ; B_{1}=\left(\begin{array}{ll}1 & 0 \\ 0 & 0\end{array}\right)$ and $B_{3}=\left(\begin{array}{ll}0 & 0 \\ 0 & 1\end{array}\right) \Rightarrow g(\lambda)=\left(\begin{array}{cc}\lambda & 1 \\ 0 & \lambda^{2}\end{array}\right)$.

If $\mathrm{h}(\lambda)=\operatorname{det}[\mathrm{f}(\lambda) \mathrm{g}(\lambda)]=A_{0}+A_{1} \lambda+A_{2} \lambda^{2}+A_{3} \lambda^{3}+A_{4} \lambda^{4}+A_{5} \lambda^{5}+A_{6} \lambda^{6}=\lambda^{3}+\lambda^{4}-\lambda^{6}$. $\operatorname{det} \mathrm{f}(\lambda)=1+\lambda-\lambda^{3}$ and $\operatorname{det} \mathrm{g}(\lambda)=\lambda^{3}$.

Let $h(A)=A^{3}-A^{4}+A^{6}=0=A^{3}\left(I+A-A^{3}\right)$

$\Rightarrow \operatorname{det} g(A)=0$ or $\operatorname{det} f(A)=0$.

Conversely,

Let $\operatorname{det} \mathrm{f}(\mathrm{A})=0=\mathrm{I}+\mathrm{A}-\mathrm{A}^{3}$ or $\operatorname{det} \mathrm{g}(\mathrm{A})=\mathrm{A}^{3}=0$.

$\Rightarrow h(A)=A^{3}-A^{4}+A^{6}=A^{3}\left(I+A-A^{3}\right)=A^{3}(0)=0$.

\section{Conclusion}

In this paper we have proved a theorem on the determinant of the product of two polynomial matrices. Similarly we can prove all results relating to matrices and their determinants.

\section{References}

[1] Raj Kumar Kanwar, ' A Generalization of the Cayley-Hamilton Theorem," Advances in PureMathematics, 2013, 3, 109-115 Published Online January 2013 (http://www.scirp.org/journal/apm).

[2] M. Buslowicz and T. Kaczorek, "Reachability and Mini-mum Energy Control of Positive Linear Discrete-Time Systems with One Delay," Proceedings of 12th Mediter-ranean Conference on Control and Automation, Kasadesi- Izmur, CD ROM, 2004.

[3] B. G. Mcrtizios and M. A. Christodolous, "On the Gener-alized Cayley-Hamilton Theorem," IEEE Transactions on Automatic Control, Vol. 31, No. 1, 1986, pp. 156-157.

[4] T. Kaczorek, "Generalization of the Cayley-Hamilton Theo-rem for Non-Square Matrices," International Conference of Fundamentals of Electronics and Circuit Theory XVIII- SPETO, Gliwice, 1995, pp. 77-83.

[5] T. Kaczorek, "Extension of the Cayley-Hamilton Theo-rem for Continuous-Time Systems with Delays," Interna-tional Journal of Applied Mathematics and Computer Sci-ence, Vol. 15, No. 2, 2005, pp. 231-234.

[6] T. Kaczorek, "An Existence of the Cayley-Hamilton Theo-rem for Singular 2-D Linear Systems with Non-Square Matrices," Bulletin of the Polish Academy of Sciences. Technical Sciences, Vol. 43, No. 1, 1995, pp. 39-48.

[7] T. Kaczorek, "Extensions of the Cayley-Hamilton Theo-rem for 2D Continuous-Discrete Linear Systems," Ap-plied Mathematics and Computation Sciences, Vol. 4, No. 4, 1994, pp. 507-515.

[8] T. Kaczorek, "Vectors and Matrices in Automation and Electrotechnics," Polish Scientific Publishers, Warsaw, 1988. 